\title{
Deep learning based pectoral muscle segmentation on Mammographic Image Analysis Society (MIAS) mammograms
}

\author{
Young Jae Kim ${ }^{1}$, Eun Young Yoo², Kwang Gi Kim ${ }^{1}$ \\ ${ }^{1}$ Department of Biomedical Engineering, Gachon University Gil Medical Center, Gachon University College of Medicine, Inchon, \\ Korea \\ ${ }^{2}$ Department of Radiology, Gachon University Gil Medical Center, Gachon University College of Medicine, Incheon, Korea
}

Received: November 23, 2020

Revised: January 3, 2021

Accepted: January 19, 2021

\section{Corresponding authors:}

Kwang Gi Kim

Department of Biomedical Engineering, Gachon University Gil Medical Center, Gachon University College of Medicine, 21 Namdong-daero 774beongil, Namdong-gu, Incheon 21565, Korea

Tel: +82-32-820-4036

E-mail:kimkg@gachon.ac.kr

Eun Young Yoo

Department of Radiology, Gachon University Gil Medical Center, Gachon University College of Medicine, 21 Namdong-daero 774beon-gil, Namdong-gu, Incheon 21565, Korea

Tel: +82-32-460-8269

E-mail: yey0227@gilhospital.com

This is an Open Access article distributed under the terms of the Creative Commons Attribution Non-Commercial License (https:// creativecommons.org/licenses/ by-nc/4.0/).

\begin{abstract}
Purpose: The purpose of this study was to propose a deep learning-based method for automated detection of the pectoral muscle, in order to reduce misdetection in a computer-aided diagnosis (CAD) system for diagnosing breast cancer in mammography. This study also aimed to assess the performance of the deep learning method for pectoral muscle detection by comparing it to an image processing-based method using the random sample consensus (RANSAC) algorithm.

Methods: Using the 322 images in the Mammographic Image Analysis Society (MIAS) database, the pectoral muscle detection model was trained with the U-Net architecture. Of the total data, $80 \%$ was allocated as training data and $20 \%$ was allocated as test data, and the performance of the deep learning model was tested by 5 -fold cross validation.

Results: The image processing-based method for pectoral muscle detection using RANSAC showed $92 \%$ detection accuracy. Using the 5 -fold cross validation, the deep learning-based method showed a mean sensitivity of $95.55 \%$, mean specificity of $99.88 \%$, mean accuracy of $99.67 \%$, and mean dice similarity coefficient of $95.88 \%$.

Conclusion: The proposed deep learning-based method of pectoral muscle detection performed better than an existing image processing-based method. In the future, by collecting data from various medical institutions and devices to further train the model and improve its reliability, we expect that this model could greatly reduce misdetection rates by CAD systems for breast cancer diagnosis.
\end{abstract}

Keywords: Deep learning; Mammography; Pectoralis muscles

\section{INTRODUCTION}

Among breast imaging methods, mammography is widely used in screening for breast cancer [1]. However, the results can be difficult to interpret for dense breasts, which leads to a high risk of misdiagnosis [2]. For this reason, there has been ongoing research on computer-aided 
diagnosis (CAD) systems, in order to reduce the number of misdiagnoses and to improve the accuracy of diagnosis by radiologists using mammography [3-5]. CAD systems use computer algorithms to enable objective and accurate detection of lesions that are difficult to distinguish with the naked eye. However, accurate lesion detection by these CAD systems can be negatively affected for various reasons. In particular, in CAD systems used to diagnose breast cancer, the pectoral muscle shows a similar pixel intensity to that of lesions of the right mediolateral oblique (RMLO) and left mediolateral oblique (LMLO) muscles, which can cause misdetection [6]. To prevent this, a separate pectoral muscle detection algorithm is required.

In 2016, we developed an image processing-based automated pectoral muscle detection algorithm using the random sample consensus (RANSAC) algorithm on images from the Mammographic Image Analysis Society (MIAS) database [7]. This algorithm had a detection accuracy of $92.2 \%$, which was higher than that found in other studies using the MIAS database [8-12]. Nevertheless, detection accuracy was poor in some images due to the complex shape of the pectoral muscle, and so the algorithm needed to be improved.

Recently, the number of researches on pectoral muscle segmentation using computer algorithms are increasing. Shen et al. [13] reported an algorithm for automatic pectoral muscle segmentation that combines a genetic algorithm and a morphological selection algorithm. As a result of verification with the Mini-MIAS database, the false positive (FP) rate was $2.03 \%$ and the false negative (FN) rate was $6.90 \%$. Gardezi et al. [14] reported an algorithm of segmenting the boundary between the breast region and the breast parenchyma using an adaptive gamma correction method. As a result, an accuracy of $98.45 \%$ and a jaccrard similarity index of 92.79\% were shown.

Recent advances in hardware have created a favorable environment for deep learning techniques, which have been applied in various fields. Convoluted neural networks (CNNs) especially, as one type of deep learning technique, have been used with outstanding results in various imaging fields $[15,16]$. In medical imaging as well, numerous studies using CNNs have reported better performance than conventional image processing techniques [17-19]. Thus, in this study, we aimed to use deep learning in pectoral muscle detection, to improve upon the previously encountered problems for the complex-shaped pectoral muscle, and to enhance detection accuracy. The deep learning model for pectoral muscle detection was trained using the same MIAS database as before, and the performance of the algorithm was assessed in comparison to the results of the image processing-based method using RANSAC.

\section{METHODS}

\section{Data}

This study used mammograms from the mini-MIAS database, which is one of several open access databases. The MIAS database consists of scans of 322 mammogram films taken as part of the United Kingdom's national breast cancer screening program [20]. All the images in the mini-MIAS database consist of RMLO or LMLO views excluding right craniocaudal and left craniocaudal, and the images sizes are 1,024 × 1,024 pixels. For annotation data to use in deep learning, regions of interest (ROIs) for the pectoral muscle were drawn manually by a radiologist. The ROIs were drawn using ImageJ software version 1.52a (National Institutes of Health, Bethesda, MD, USA). In the mediolateral oblique images, polygonal ROIs were drawn by distinguishing the boundary between the breast region and the breast parenchyma. A binary mask was made, using 0 for outside the ROI and 255 for inside the ROI, and this was used as the ground truth. Fig. 1 shows one of the binary mask images used as the ground truth.

The size of the data set used in this study was not large, and so 5 -fold cross validation was used to ensure that the model would be robust in terms of data dependency. For each cross validation, $80 \%$ of the overall data ( 257 to 258 scans) was used as learning data, and 20\% (64 to 65 scans) was separately constructed and used as test data. Each data point was used exactly once as test data, without duplication.

\section{Development environment}

The system for deep learning consisted of 4 NVIDIA TITAN Xp (NVIDIA Corp., Santa Clara, CA, USA) graphics processing
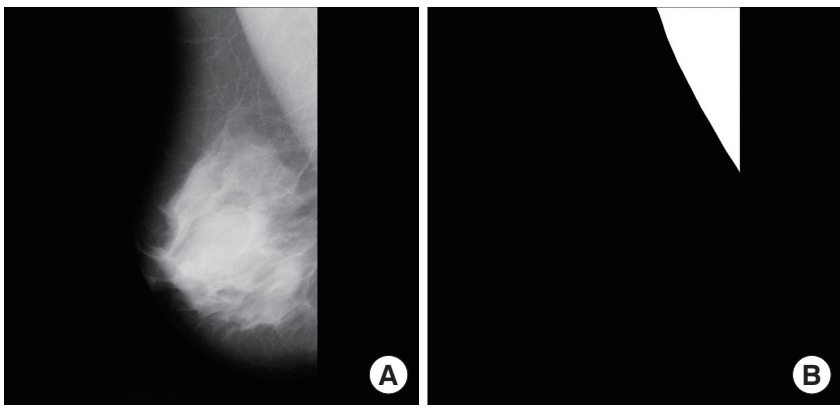

Fig. 1. Examples of an image from the Mammographic Image Analysis Society (MIAS) database (A) and annotation data made directly by a specialist (B). 
units, a Xeon E5-1650 v4 (Intel Corp., Santa Clara, CA, USA) central processing unit, and $128 \mathrm{~GB}$ of random access memory (RAM). Deep learning was conducted using Python 2.7.6 and the Keras 2.1.5 framework (Python Software Foundation, Wilmington, DE, USA) with a TensorFlow backend in the Ubuntu 14.04 operating system (Canonical Ltd., London, UK).

\section{Data augmentation}

The images from the MIAS database used in this experiment were insufficient to train the deep learning model. Data augmentation was performed to acquire a sufficient quantity of learning data [21]. An arbitrary combination of flips, rotations, translations, and stacking were used to expand the learning data set 20 -fold.

\section{Training the deep learning model}

For the CNN in this study, the U-Net model was used. One advantage of the U-Net model is that it has a structure that re-uses encoding and decoding phases via skip connections (Fig. 2); as a result, when images are reconstructed using the network, the original image can be reconstructed without losing even fine details, meaning that the output images have excellent quality $[22,23]$. The U-Net architecture was composed of 23 layers, the number of parameters used was $5,142,433$, and the Jaccard loss function was used. For the learning environment, the batch size was set to 8 , and the number of epochs was fixed to 300 based on an Adam optimizer. The learning rate was set to 0.001 up to epoch 100 ,
0.0001 from epoch 100 to 250 , and 0.00001 from epoch 250 to 300 .

\section{Ethical statement}

Institutional Review Board of Gachon University Gil Medical Center approval and signed informed consent were not needed, as the entire study was performed with medical images included in public databases.

\section{RESULTS}

In this study, deep learning was used to train a model to detect pectoral muscle in MIAS database images. The trained model was applied to separately constructed test data to assess its performance. Fig. 3 compares the ground truth data from the test data with the results automatically extracted using the trained model.

For each cross validation, the model was tested using four statistical indices: sensitivity, specificity, accuracy, and dice similarity coefficient (DSC). The extracted results of the deeplearning model were compared pixel-by-pixel with the ground truth data, the true positive (TP), FP, true negative, and FN rates were calculated, and the statistical indices were calculated using the equations below. From the results of the five cross validations, the mean sensitivity was $95.55 \%$, the mean specificity was $99.88 \%$, the mean accuracy was $99.67 \%$, and the mean DSC was $95.88 \%$ (Table 1 ).

The deep learning-based pectoral muscle detection algo-
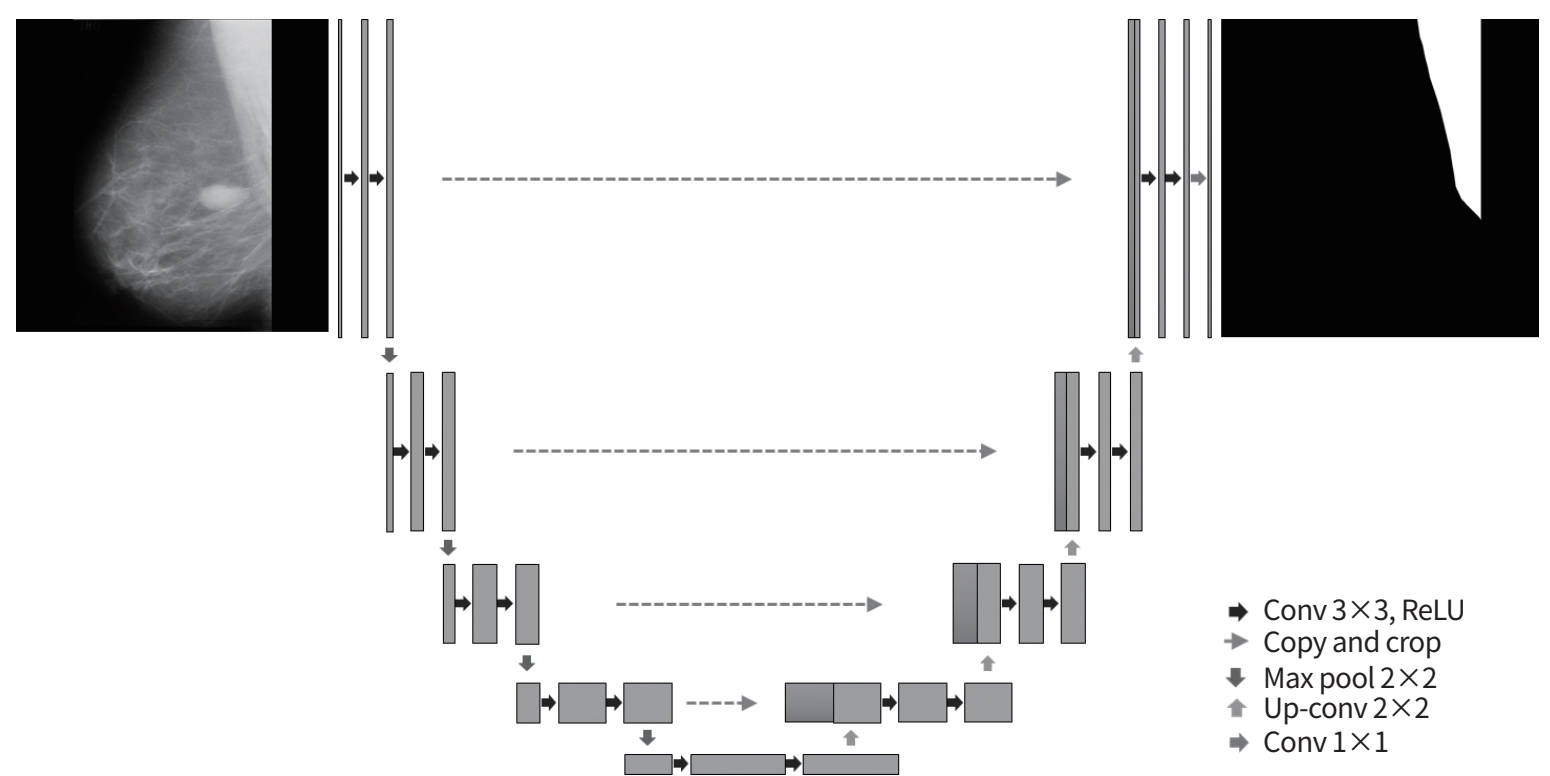

Fig. 2. The U-Net architecture consisting of encoding and decoding units. conv, convolution; ReLU, rectified linear unit. 

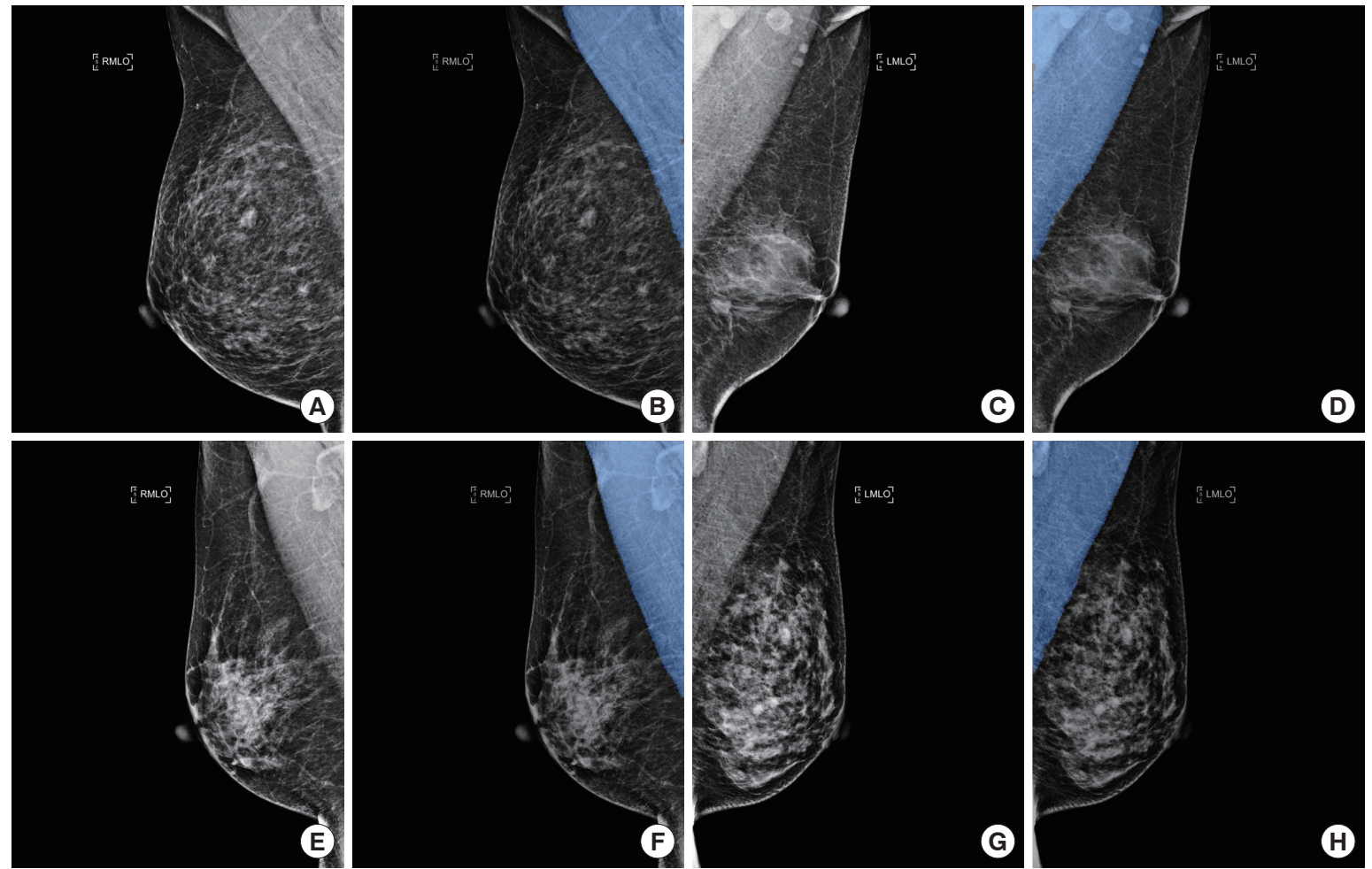

Fig. 3. Examples of pectoral muscle detection results from the test data: (A, C, E, G) original image, (B, D, F, H) deep learning-based automated detection results. RMLO, right mediolateral oblique; LMLO, left mediolateral oblique.

Table 1. Results of 5-fold cross validation for the deep learningbased pectoral muscle detection method

\begin{tabular}{lcccc}
\hline & $\begin{array}{c}\text { Sensitivity } \\
(\%)\end{array}$ & $\begin{array}{c}\text { Specificity } \\
(\%)\end{array}$ & $\begin{array}{c}\text { Accuracy } \\
(\%)\end{array}$ & $\begin{array}{c}\text { DSC } \\
(\%)\end{array}$ \\
\hline CV1 & 94.01 & 99.91 & 99.57 & 94.50 \\
CV2 & 96.40 & 99.87 & 99.68 & 96.78 \\
CV3 & 95.65 & 99.90 & 99.72 & 96.45 \\
CV4 & 95.97 & 99.83 & 99.66 & 96.18 \\
CV5 & 95.73 & 99.89 & 99.71 & 95.49 \\
Total & 95.55 & 99.88 & 99.67 & 95.88
\end{tabular}

DCS, dice similarity coefficient; CV, cross validation.

Table 2. Comparison of performance (categorical) between the deep learning-based pectoral muscle detection method and the image processing-based method using RANSAC

\begin{tabular}{lccc}
\hline & Good & Acceptable & Unacceptable \\
\hline RANSAC method & 264 & 36 & 22 \\
Deep learning method & 322 & 0 & 0 \\
\hline
\end{tabular}

RANSAC, random sample consensus.

rithm was assessed using the same method as our previous study on an image processing-based method using the RANSAC algorithm, and the results of the two models were
Table 3. Comparison of performance (detection accuracy) between the deep learning-based pectoral muscle detection method and the image processing-based method using RANSAC

\begin{tabular}{lcc}
\hline Category & $\begin{array}{c}\text { RANSAC } \\
\text { method (\%) }\end{array}$ & $\begin{array}{c}\text { Deep learning } \\
\text { method (\%) }\end{array}$ \\
\hline FP & $4.51 \pm 6.53$ & $2.88 \pm 6.05$ \\
FN & $5.68 \pm 8.57$ & $4.27 \pm 8.72$ \\
FP $<5 \%, F N<5 \%$ & 56.5 & 71.0 \\
$5 \%<$ FP $<15 \%, 5 \%<$ FN $<15 \%$ & 31.5 & 20.7 \\
$15 \%<$ FP, $15 \%<$ FN & 12.0 & 8.3 \\
\hline
\end{tabular}

Values are presented as mean \pm standard deviation.

RANSAC, random sample consensus; FP, false positive; FN, false negative.

compared. We assessed the differences between the automated detection results of the deep learning model and the manually drawn ground truth data. Concordance $\geq 90 \%$ between the deep learning-based automated detection and the manual detection images was defined as "good," concordance $\geq 50 \%$ and $<90 \%$ was defined as "acceptable," and concordance $<50 \%$ was defined as "unacceptable" [7]. The previous method using the RANSAC algorithm showed 264 "good" results, whereas the deep learning model showed 
322 "good" results (Table 2). The FP and FN rates of the previous method were, respectively, $4.51 \% \pm 6.53 \%$ and $5.68 \% \pm$ $8.57 \%$ (Table 3). In contrast, the FP and FN rates of the deep learning method were, respectively, $2.88 \% \pm 6.05 \%$ and $4.27 \%$ $\pm 8.72 \%$.

\section{DISCUSSION}

This study proposed a pectoral muscle detection method using deep learning with the MIAS database. Although the proposed method used all the images in the MIAS database, there was too little data to construct a separate validation set. We used 5-fold cross validation to supplement the shortage of validation data and to minimize the data dependency of the model. The proposed model showed high accuracy, with a mean sensitivity of $95.55 \%$ and mean DSC of $95.88 \%$.

We also compared the results of the deep learning-based pectoral muscle detection method with a previous image processing-based pectoral muscle detection method using the RANSAC algorithm. While the RANSAC method showed "unacceptable" results for 22 images, the deep learning method did not show "unacceptable" results for even a single image. Moreover, when the misdetection rate was inspected, the RANSAC algorithm showed FP and FN rates $<5 \%$ for only $56.5 \%$ of the images, whereas the deep learning algorithm showed a higher proportion of images (71.0\%) with FP and $\mathrm{FN}$ rates $<5 \%$. These results demonstrate that the deep learning algorithm achieved more accurate and more stable detection results than the RANSAC algorithm.

In image processing, RANSAC is an algorithm for approximation. Approximation cannot guarantee the detection of an accurate pectoral muscle region. Although there have been attempts to approximate the area of a curved pectoral muscle using nonlinear RANSAC, it is quite difficult to accurate approximate the pectoral muscle. This weakness is thought to have led to the 22 "unacceptable" results. In contrast, because deep learning makes an overall judgment about the shape and the relationship attenuation and pixels based on the training images, it requires generalized, diverse training data. In this study, we used the same data set in order to compare the new model objectively with the previous image processing-based pectoral muscle detection algorithm. The data set was somewhat small to train a deep learning model. The fact that we were still able to obtain relatively good results is thought to be because the shape and position of the pectoral muscle was fairly consistent, and did not differ greatly between patients. Although the possibility of overfit- ting can be suspected, these doubts can be partially resolved based on the results of cross validation.

This study had some limitations. In current clinical settings, digital mammography is used in most instances, but the MIAS database contains data obtained by scanning film mammograms. Therefore, there is no guarantee that a deep learning model trained on MIAS data will show good results for digital mammograms. In order to resolve this issue, it will be necessary to collect more digital mammograms, and to further train a deep learning model based on the collected data. It will also be important to collect data from diverse medical institutions and devices, and to objectively validate the deep learning model through multi-center validation. We expect that these additional studies would further enhance the clinical reliability of the deep learning-based pectoral muscle detection method proposed in this report.

In conclusion, the experiments in this study demonstrated that the deep learning-based pectoral muscle detection method achieved a more accurate and stable detection rate than a previous image processing-based method. We expect that this type of deep learning technique could be useful to overcome the limitations of conventional image processing techniques.

\section{CONFLICTS OF INTEREST}

No potential conflict of interest relevant to this article was reported.

\section{ACKNOWLEDGMENTS}

This work was supported by the Gachon University (20180669) and Gil Medical Center (Grant number: 2018-5299).

\section{ORCID}

Young Jae Kim https://orcid.org/0000-0003-0443-0051

Eun Young Yoo https://orcid.org/0000-0002-7849-2477

Kwang Gi Kim https://orcid.org/0000-0001-9714-6038

\section{AUTHOR CONTRIBUTIONS}

Conception or design: EYY, KGK.

Acquisition, analysis, or interpretation of data: YJK, EYY, KGK.

Drafting the work or revising: YJK.

Final approval of the manuscript: EY, KGK. 
PRECISION AND FUTURE MIEDICINE

Pectoral muscle segmentation on mammograms

\section{REFERENCES}

1. Lee CH, Dershaw DD, Kopans D, Evans P, Monsees B, Monticciolo $D$, et al. Breast cancer screening with imaging: recommendations from the Society of Breast Imaging and the ACR on the use of mammography, breast MRI, breast ultrasound, and other technologies for the detection of clinically occult breast cancer. J Am Coll Radiol 2010;7:18-27.

2. Boyd NF, Guo H, Martin LJ, Sun L, Stone J, Fishell E, et al. Mammographic density and the risk and detection of breast cancer. N Engl J Med 2007;356:227-36.

3. Park BE, Jang WS, Yoo SK. Texture analysis of supraspinatus ultrasound image for computer aided diagnostic system. Healthc Inform Res 2016;22:299-304.

4. Subashini TS, Ramalingam V, Palanivel S. Pectoral muscle removal and detection of masses in digital mammogram using CCL. Int J Comput Appl 2010;1:71-6.

5. van Ginneken B, Schaefer-Prokop CM, Prokop M. Computer-aided diagnosis: how to move from the laboratory to the clinic. Radiology 2011;261:719-32.

6. Vaidehi K, Subashini TS. Automatic identification and elimination of pectoral muscle in digital mammograms. Int J Comput Appl 2013;75:15-8.

7. Yoon WB, Oh JE, Chae EY, Kim HH, Lee SY, Kim KG. Automatic detection of pectoral muscle region for computer-aided diagnosis using MIAS mammograms. Biomed Res Int 2016;2016:5967580.

8. Alam N, Islam MJ. Pectoral muscle elimination on mammogram using K-means clustering approach. Int J Comput Vis Signal Process 2014;4:11-21.

9. Mustra M, Grgic M. Robust automatic breast and pectoral muscle segmentation from scanned mammograms. Signal Process 2013;93:2817-27.

10. Molinara M, Marrocco C, Tortorella F. Automatic segmentation of the pectoral muscle in mediolateral oblique mammograms. Proceedings of the 26th IEEE International Symposium on Computer-Based Medical Systems; 2013 Jun 20-22; Porto, PT. Piscataway (NJ): IEEE; 2013. p. 506-9.

11. Kwok SM, Chandrasekhar R, Attikiouzel Y. Automatic pectoral muscle segmentation on mammograms by straight line estimation and cliff detection. The Seventh Australian and New Zealand Intelligent Information Systems Conference; 2001 Nov 18-21; Perth, AU. Piscataway (NJ): IEEE; 2002. p. 67-72.
12. Raba D, Oliver A, Marti J, Peracaula M, Espunya J. Breast segmentation with pectoral muscle suppression on digital mammograms. Lectu Notes Comput Sci 2005;3523: 471-8.

13. Shen R, Yan K, Xiao F, Chang J, Jiang C, Zhou K. Automatic pectoral muscle region segmentation in mammograms using genetic algorithm and morphological selection. J Digit Imaging 2018;31:680-91.

14. Gardezi SJS, Adjed F, Faye I, Kamel N, Eltoukhy MM. Segmentation of pectoral muscle using the adaptive gamma corrections. Multimed Tools Appl 2018;77:3919-40.

15. Poedjiastoeti W, Suebnukarn S. Application of convolutional neural network in the diagnosis of jaw tumors. Healthc Inform Res 2018;24:236-41.

16. Jeon E, Kim Y, Park H, Park RW, Shin H, Park HA. Analysis of adverse drug reactions identified in nursing notes using reinforcement learning. Healthc Inform Res 2020;26: 104-11.

17. Suzuki K. Overview of deep learning in medical imaging. Radiol Phys Technol 2017;10:257-73.

18. Cheng JZ, Ni D, Chou YH, Qin J, Tiu CM, Chang YC, et al. Computer-aided diagnosis with deep learning architecture: applications to breast lesions in US images and pulmonary nodules in CT scans. Sci Rep 2016;6:24454.

19. Hua KL, Hsu CH, Hidayati SC, Cheng WH, Chen YJ. Computer-aided classification of lung nodules on computed tomography images via deep learning technique. Onco Targets Ther 2015;8:2015-22.

20. Matheus BR, Schiabel H. Online mammographic images database for development and comparison of CAD schemes. J Digit Imaging 2011;24:500-6.

21. Perez L, Wang J. The effectiveness of data augmentation in image classification using deep learning. arXiv 2017 Dec 13. https://arxiv.org/abs/1712.04621.

22. Norman B, Pedoia V, Majumdar S. Use of 2D U-Net convolutional neural networks for automated cartilage and meniscus segmentation of knee MR imaging data to determine relaxometry and morphometry. Radiology 2018; 288:177-85.

23. Ronneberger O, Fischer P, Brox T. U-Net: convolutional networks for biomedical image segmentation. In: Navab $\mathrm{N}$, Hornegger J, Wells W, Frangi A. editors. International Conference on Medical Image Computing and Computer-assisted Intervention; 2015 Oct 5-9; Munich, DE. Cham (CH): Springer; 2015. p. 234-41. 